\title{
Histologische und Histochemische Untersuchungen über das Leichenwachs
}

\author{
Kinshi Mitsuki* \\ Aus dem Pathologischen Institut der Universität Tohoku, Sendai, \\ und der Medizinischen Klinik des Zentralkrankenhauses von Akita
}

(Received for publication, September 20, 1961)

\section{EINLEITUNG}

Das Problem der Entstehungsvorgänge des Leichenwachses, das von jeher eine Anzahl von Forschern interessiert, ist durch experimentelle Untersuchungen zu einem gewissen Abschluß gekommen. Die Substanzen, die das Leichenwachs bilden, wurden schon seit langem von Biochemikern untersucht, und in den letzten Jahren sind aussichtsreiche Versuche in dieser Richtung ausgeführt worden. Dagegen fehlt bis heute noch eine eingehende und ausführliche histochemische Forschung des Leichenwachses. Der Verfasser hat mit Heranziehung histochemischer Methoden ein Leichenwachs untersucht, das in unserem pathologischen Institut aufbewahrt ist. Das Resultat schien mir umso interessanter, da es mit den Ergebnissen in vitro von Biochemikern übereinstimmte. Der Verfasser wurde dadurch zur folgenden Mitteilung veranlaßt.

\section{MATERIAL}

Zu meinen Untersuchungen stand ein Leichenwachs zur Verfügung, das im Mai 1922 aus dem Friedhof eines buddhistischen Tempels in SEndaI zufällig ausgegraben worden und seither in unserem pathologischen Institut aufbewahrt war. Es war das Leichenwachs einer Patientin, die in 1905 in der Schwangerschaft wegen Beriberi gestorben war. Der Sarg war an der Grenze von verfaulter Erde und Lehmzone vergraben und wurde durch reichliches hartes Wasser gespült. Nach der Ausgrabung bleibt das Leichenwachs bis heute in einem Glaskasten in trockenem Zustand erhalten. Obzwar das Gesicht anscheinend ziemlich verformt ist, sind die Augengruben, die Ohrmuscheln, die Augenbrauen und die Zähne noch jetzt als solche gut zu erkennen. Die Brust- und Bauchhöhlen bilden

*)参木 錦 司

Diese Arbeit wurde in 1936 unter der Leitung vom damaligen Direktor des Pathologischen Instituts, von Herm Professor Dr. O. KImura ausgeführt. Der Verfasser ist ihm für seine Anregung und Leitung zum herzlichsten Dank verpflichtet. 
einen gemeinsamen Hohlraum, sodaß die einzelnen Organe, abgesehen von einem kleinen Teil des Zwerchfells, der Rippen und der Nieren, nicht mehr erkennbar sind. Zu meinem Experiment wurden aus diesem Leichenwachs sieben kleine Muskelstücke mit subkutanem Fettgewebe von verschiedenen Körperteilen und Stücke der Haut und der Niere, die nämlich das einzige erkennbare Organ war, ausgeschnitten.

Aus derselben Stelle, wo das oben erwähnte Leichenwachs gefunden wurde, wurde ein anderes Leichenwachs gleichzeitig ausgegraben. Aus dem letzteren konnte ich zu meiner Untersuchung nur das Gehirn und den Knochen benutzen. Die Organe waren in Formalin und ORTHscher Losung konserviert.

\section{UNTERSUCHUNGSMETHODEN}

Kleine Stücke aus dem Leichenwachs wurden 2 oder 3 Tage in fliessendem Wasser sorgfältig gewaschen. Ein Teil des Materials wurde danach sofort in Gelatin eingebettet. Der andere Teil wurde 5 Tage in mit Calcium salicylicum gesättigter $10 \%$ Formalinlösung gelegt. Nach dem Verfahren wurde er ebenfalls in Gelatin eingebettet. Zur histologischen Untersuchung wurden Gefrierschnitte angefertigt. Die kleinen Gewebsstücke in Gelatin wurden in $5 \%$ Formalinlösung konserviert, in einem dunkelen Zimmer aufbewahrt und je nach Bedarf zur weiteren Untersuchung benutzt. Ich habe dabei nicht nur die üblichen Färbungen (Hämatoxylin-Eosin und van Gieson), sondern auch spezifische Lipoidfärbungen (Nilblausulfat-, Sudan III-, Osmiumsäure- und Kresylechtviolettfärbungen, sowie Färbungen von Fischler, Smith-Dietrich und Ciaccio) angewandt. Ferner wurde der Kalk durch Grandis-Maini's Purpurin-Färbung, Kossa's Silbermethode, RoEHLsche und Schmorlsche Färbungen und durch Na. alizarin-sulforicum nachgewiesen. Weiter habe ich doppelbrechende Substanzen im Gewebe, ihren optischen Charakter und ihr Verhalten in Bezug auf die Temperatur untersucht. Quantitative Bestimmung von Cholesterin im Muskelgewebe wurde nach der Methode von BLoor, die Darstellung von Cholesterinlecithin nach der Methode von A. Schultze und Cholesterinnachweis im Gewebe nach GoLoDETzscher Reaktion vorgenommen. In der Beurteilung der Ergebnisse der Fettfärbungen habe ich fast ausschließlich auf KaWAmuRA's Gruppenreaktion Bezug genommen.

\section{RESULTATE DER HISTOLOGISCHEN UNTERSUCHUNGEN}

1. Knochengewebe

Die Knochengrundsubstanzen und Knochenplatten waren relativ gut erhalten und durch Hämatoxylin-Eosin-Färbung als solche leicht darzustellen. Die Knochenkörperchen waren mit Hämatoxylin intensiv gefärbt. In den Markhøhlen fanden sich zahlreiche, strukturlose und mit Hämatoxylin stark gefärbte Nadelkristalle vor. Seltener waren Strukturen zu finden, die als 
K. Mitsuki

Zellen und Kerne aufzufassen waren. Hie und da fanden sich fettgewebesartige Spalten. An der Stelle des Periostes sammelten sich zahlreiche, radiär angeordnete Nadelkristalle, und große Menge strukturloser, mit Hämatoxylin intensiv gefärbter Substanz. Die Fettfärbungen ergaben folgende Resultate.

1) Durch Nilblausulfat wurde der Knochen nicht dargestellt, und das Knochenmark war blau gefärbt.

2) Durch Sudan III war der Knochen leicht bräunlich, das Knochenmark gelblichrot gefärbt. Im Knochenmark fanden sich granulierte, rotliche Substanzen. Die Reaktionen nach Smith-Dietrich und nach Ciaccio waren im Knochenmark positiv, dagegen gab Osmiumsäure keine I. und II. Reaktion. Kresylechtviolett gab nur schwache positive Färbung. Eine zusammenfassende Darstellung findet man in Tabelle I. Ferner habe ich die Darstellung von

Tabelle I

\begin{tabular}{|c|c|c|c|}
\hline \multicolumn{2}{|c|}{ Färbungsmethode } & Knochen & Knochenmark \\
\hline \multicolumn{2}{|l|}{ Nilblausulfat } & ungefärbt & blau \\
\hline \multicolumn{2}{|l|}{ Sudan III } & $\begin{array}{l}\text { schwach } \\
\text { blau }\end{array}$ & $\begin{array}{l}\text { gelblichrot } \\
\text { punktförmig rot }\end{array}$ \\
\hline \multicolumn{2}{|l|}{ Fischler } & + & \pm \\
\hline \multicolumn{2}{|l|}{ Smith-Dietrich } & - & + \\
\hline \multicolumn{2}{|l|}{ Ciaccio } & - & + \\
\hline \multirow{2}{*}{ Osmiumsäure } & I-Reak. & - & - \\
\hline & II-Reak. & - & - \\
\hline \multicolumn{2}{|c|}{ Kresylechytiolett } & - & \pm \\
\hline \multicolumn{2}{|l|}{ Purpurin } & + & \pm \\
\hline \multicolumn{2}{|l|}{ Kóssa } & + & + \\
\hline \multicolumn{2}{|l|}{ Schmorl } & + & + \\
\hline \multicolumn{2}{|c|}{ Nat. alizarinsulforicum } & + & + \\
\hline \multicolumn{2}{|l|}{ Roehl } & + & - \\
\hline \multicolumn{2}{|c|}{ A. Schultze'sche Reak. } & - & - \\
\hline \multicolumn{2}{|c|}{ Golodetz'sche Reak. } & - & - \\
\hline \multicolumn{2}{|c|}{$\begin{array}{l}\text { Durch Aetheralkohol mit Zusatz von } \\
\text { Salzsäure nach Fischler. (Aetheralkohol : } \\
\text { Salzsåure }=100: 1 \text { ) }\end{array}$} & & entfärbt \\
\hline \multicolumn{2}{|c|}{$\begin{array}{l}\text { Durch } 1 \% \text { wässerige Salzsäurelösung nach } \\
\text { Fischler }\end{array}$} & & $\begin{array}{l}\text { zum Teil } \\
\text { entfärbt }\end{array}$ \\
\hline
\end{tabular}


Kalksalz im Knochen und Knochenmark versucht. A. Schultzesche und GoLodETzsche Reaktionen waren negativ. Mehrere Schnitte wurden nach FISCHLER mit Kupferazetat behandelt und entweder in $1 \%$ wässeriger Salzsäurelosung oder in Ätheralkoholgemisch mit Salzsäure gelegt. Wenn vorhin positive Hämatoxylinfärbung nach Behandlung mit wässeriger Salzsäure negativ ausfällt, so ist die Reaktion auf Kalk zurückzuführen. Wenn die Reaktion an mit wässoriger Salzsäurelösung entkalkten Schnitten positiv ausfällt und erst nach Behandlung mit Ätheralkohol negativ umschlägt, so liegen Fettsäuren vor, weil fettsaurer Kalk in Ätheralkoholgemisch mit Salzsäure löslich ist. Nach meinen Beobachtungen waren im Knochenmark unterschiedliche Menge fettsaurer Seife und fettsauren Kalks und geringe Menge Phosphatid vorhanden.

2. Gehirn

Das Hirngewebe ließ durch Hämatoxylin-Eosin-Färbung im allgemeinen keine eigentliche Struktur erkennen. Die Gliafasern waren dagegen relativ gut darzustellen. Man konnte sogar manchmal Umrisse der Ganglienzellen feststellen. Überall fanden sich radiär angeordnete Schattenfiguren von Cholesterintafeln in fibrillären Strukturen. Hin und wieder kamen, nach BiELschowskischer Färbung, spaltförmige oder ringformige Strukturen vor, die als Gefäße aufgefaßt wurden. Die Neurofibrillen waren gar nicht nachweisbar. Das Hirngewebe war durch Nilblausulfat diffus blau gefärbt, und hie und da fanden sich fleckweise rotlichblau gefärbte Herdchen. Durch Sudan III färbte sich das Gewebe diffus schwach gelblichrot, mit zerstreuten fleckweisen orangenroten

TABELLE II

\begin{tabular}{|c|c|}
\hline Färbungsmethode & Hirnsubstanz \\
\hline Nilblausulfat & $\begin{array}{l}\text { diffus blau, punktförmig rötlich- } \\
\text { blau }\end{array}$ \\
\hline Sudan III & $\begin{array}{l}\text { diffus schwach gelblichrot, } \\
\text { fleckweise orangenrot }\end{array}$ \\
\hline Fischler & - \\
\hline Smith-Dietrich & + \\
\hline Ciaccio & + \\
\hline \multirow{2}{*}{ Osmiumsaüre } & + \\
\hline & - \\
\hline Kresylechtviolett & $\mathrm{HH}$ \\
\hline Purpurin & \pm \\
\hline Kóssa & \pm \\
\hline Schmorl & - \\
\hline Nat. alizarinsulforicum & + \\
\hline Roehl & \pm \\
\hline A. Schultze'sche Reak. & + \\
\hline Golodetz'sche Reak. & - \\
\hline
\end{tabular}


Herdchen. Die Reaktionen auf Lipoidsubstanzen nach Frschler, SmithDietrich, Ciaccio usw., die I. Osmiumsäurereaktion und Kresylechtviolettfärbung waren stark positiv. Nach Gruppenbestimmung von KaWamora bestehen die im Hirngewebe enthaltenen Substanzen hauptsächlich aus Phosphatid und zum kleinen Teil aus Myelin und Cholesterinlecithin. Die Ergebnisse der Untersuchung sind in Tabelle II gezeigt.

Weiter wurde das Hirngewebe auf Kalksalz geprüft. Das Resultat zeigte, daß in der Hirnsubstanz wenigstens teilweise geringe Menge Kalksalz und Phosphatkalk vorhanden war. Durch A. Schultzesche Reaktion wurde Cholesterinlecithin positiv nachgewiesen.

3. Muskelgewebe

Sieben kleine Muskelstücke wurden aus verschiedenen Stellen vom Leichenwachs ausgeschnitten. Nach gründlichem Auswaschen in fliessendem Wasser wurden vier Muskelstücke davon sofort in Gelatin eingebettet und an Gefrierschnitten untersucht. Die Muskelpräparate sind hier Muskel (C), (D), (E) und (F) genannt. Die anderen Muskelstücke wurden, nachdem sie 5 Tage in 10\% Formalinlosung blieben, die mit Calcium salicylicum gesättigt war, in Gelatin eingebettet und ebenfalls an Gefrierschnitten untersucht. Die Schnitte aus dieser Gruppe sind beziehungsweise als Muskel (K), (L) und (M) bezeichnet. Die genannte Behandlung diente zum Nachweis von fettsauer Seife im Muskelgewebe. Durch Hämatoxylin-Eosin-Färbung konnte man gar keine Muskelfaser, sondern nur strukturlose Substanzen erkennen, in denen zahlreiche, radiär angeordnete Nadelkristalle zu sehen waren. Das Bindegewebe war durch van GiesonFärbung gut darzustellen. In die Einzelheiten über nachgewiesene Fettarten und Kalksalze wird hier nicht eingegangen. Die Resultate sind aus Tabelle III ersichtlich. Was die Natur der Fettsubstanzen im Muskelgewebe anbetrifft, kam ich an Hand der Gruppenreaktionen von KawamuRA zum Schluß, daß sie im allgemeinen aus komplizierten Mischungen entweder von Cholesterin und Kephalin, Phosphatiden, Cholesterinestern, fettsauren Seifen, oder von Cholesterin und Fettsäure (Oleinsäure) bestehen.

4. Niere

Makroskopisch schien die Niere fast vollständig durch zystische Hohlräume ersetzt zu sein, sodaß das Nierenparenchym nur $1 / 3 \mathrm{~cm}$ breit war. Die Oberfläche war bräunlichgelb, und die Struktur der Niere war, außer in ihrem oberflächlichen Teil, nicht mehr erkennbar. Durch mikroskospische Untersuchung konstatierte man in mit Hämatoxylin-Eosin intensiv gefärbter strukturloser Substanz zahlreiche, radiär angeordnete Nadelkristalle und ziemlich erweiterte, gelblichbräunliche, durchsichtige und ringförmige Gebilde, die an die Arterien erinnernde Bilder darboten. Das Bindegewebe war fast gar nicht erkennbar. Durch Nilblausulfat war die Rindensubstanz rŏtlich, die Marksubstanz blieb ungefärbt. 
Tabelle III

\begin{tabular}{|c|c|c|c|c|c|c|c|c|}
\hline \multicolumn{2}{|c|}{ Färbungsmethode } & $\begin{array}{l}\text { Muskel } \\
\text { (C) }\end{array}$ & $\begin{array}{l}\text { Muskel } \\
\text { (D) }\end{array}$ & $\begin{array}{l}\text { Muskel } \\
\text { (E) }\end{array}$ & $\begin{array}{l}\text { Muskel } \\
\text { (F) }\end{array}$ & $\underset{(\mathrm{K})}{\text { Muskel }}$ & $\begin{array}{c}\text { Muskel } \\
\text { (L) }\end{array}$ & $\underset{(\mathrm{M})}{\text { Muskel }}$ \\
\hline \multicolumn{2}{|c|}{ Nilblausulfat } & $\begin{array}{l}\text { z.T. blau- } \\
\text { rötlich, } \\
\text { z.T. blau }\end{array}$ & \begin{tabular}{|l|} 
diffus \\
blau, z.T. \\
gelblich
\end{tabular} & $\begin{array}{l}\text { diffus } \\
\text { blau, z.T. } \\
\text { rötlich- } \\
\text { blau }\end{array}$ & $\begin{array}{l}\text { rötlich- } \\
\text { blau }\end{array}$ & $\begin{array}{l}\text { z.T. blau- } \\
\text { rötlich, } \\
\text { z.T. blau }\end{array}$ & im allg. & $\begin{array}{l}\text { blau- } \\
\text { rötlich, } \\
\text { z.T. gelb- } \\
\text { lich } \\
\end{array}$ \\
\hline \multicolumn{2}{|l|}{ Sudan III } & $\begin{array}{l}\text { schwach } \\
\text { gelblich- } \\
\text { rot }\end{array}$ & $\begin{array}{l}\text { rötlich u. } \\
\text { gelblich- } \\
\text { rot }\end{array}$ & $\begin{array}{l}\text { diffus } \\
\text { schwach } \\
\text { gelb- } \\
\text { lichrot }\end{array}$ & rötlich & $\begin{array}{l}\text { rötlich- } \\
\text { gelb }\end{array}$ & $\begin{array}{l}\text { gelb- } \\
\text { lichrot }\end{array}$ & $\begin{array}{l}\text { gelblich- } \\
\text { rot }\end{array}$ \\
\hline \multicolumn{2}{|l|}{ Fischler } & - & \pm & - & - & + & + & $\begin{array}{l}\text { z.T.(-) } \\
\text { z.T. }(+)\end{array}$ \\
\hline \multicolumn{2}{|c|}{ Smith-Dietrich } & \pm & + & + & \pm & + & + & $\begin{array}{l}\text { z.T.(-) } \\
\text { z.T. }(+)\end{array}$ \\
\hline \multicolumn{2}{|l|}{ Ciaccio } & + & + & + & + & + & + & \# \\
\hline \multirow{2}{*}{$\begin{array}{l}\text { Osmium- } \\
\text { säure }\end{array}$} & I-Reak. & + & \pm & + & - & - & + & $H$ \\
\hline & II-Reak. & & - & & - & - & & \\
\hline \multicolumn{2}{|c|}{ Kresylechtviolett } & + & \pm & \pm & - & \pm & - & - \\
\hline \multicolumn{2}{|l|}{ Purpurin } & H & \pm & + & + & \pm & + & + \\
\hline \multicolumn{2}{|l|}{ Kóssa } & H & + & + & + & m & H & H \\
\hline \multicolumn{2}{|l|}{ Schmorl } & + & + & + & + & \pm & + & + \\
\hline \multicolumn{2}{|c|}{$\begin{array}{c}\text { Nat. } \\
\text { alizarinsulforicum }\end{array}$} & $\mathrm{H}$ & + & + & + & + & + & + \\
\hline \multicolumn{2}{|l|}{ Roehl } & \pm & + & + & + & + & - & \pm \\
\hline \multicolumn{2}{|c|}{ A. Schultze'sche Reak. } & - & - & + & + & - & - & - \\
\hline \multicolumn{2}{|c|}{ Golodetz'sche Reak. } & - & - & - & - & - & - & - \\
\hline \multicolumn{2}{|c|}{$\begin{array}{l}\text { Durch Aetheralkohol } \\
\text { mit Zusatz von } \\
\text { Salzsäure nach Fisch- } \\
\text { ler (Aetheralkohol } \\
\text { : Salzsäure=100:1) }\end{array}$} & & & & & entfärbt & entfärbt & entfärbt \\
\hline \multicolumn{2}{|c|}{$\begin{array}{l}\text { Durch } 1 \% \text { wässerige } \\
\text { Salzsäurelösung nach } \\
\text { Fischler }\end{array}$} & & & & & $\begin{array}{l}\text { nicht } \\
\text { entfärbt }\end{array}$ & $\mid \begin{array}{c}\text { nicht } \\
\text { entfärbt }\end{array}$ & $\begin{array}{l}\text { nicht } \\
\text { entfärbt }\end{array}$ \\
\hline
\end{tabular}

Durch Sudan III war die Rindensubstanz gelblichrot, und die Marksubstanz schwach gelblichrot. Die Reaktionen nach Fischler, nach Sмiтh-Dietrich und nach CiAccio fielen positiv aus. Die I . und II. Reaktionen der Osmiumsäure waren negativ. Mit Kresylechtviolett ließ sich das Gewebe nicht färben. Die Reaktionen auf Kalksalz fielen meistens positiv aus. Die Resultate der 
Untersuchungen sind aus der Tabelle IV ersichtlich. Die Lipoidarten im Nierengewebe bestehen somit aus Gemisch geringer Menge Cholesterin und von

Tabelle IV

\begin{tabular}{|c|c|c|c|}
\hline \multicolumn{2}{|c|}{ Färbungsmethode } & Rinde & Mark \\
\hline \multicolumn{2}{|l|}{ Nilblausulfat } & schwach rötlich & ungefärbt \\
\hline \multicolumn{2}{|l|}{ Sudan III } & gelblichrot & schwach gelblich \\
\hline \multicolumn{2}{|l|}{ Fischler } & + & + \\
\hline \multicolumn{2}{|l|}{ Smith-Dietrich } & + & + \\
\hline \multicolumn{2}{|l|}{ Ciaccio } & + & + \\
\hline \multirow{2}{*}{ Osmiumsäure } & I-Reak. & - & - \\
\hline & II-Reak. & - & - \\
\hline \multicolumn{2}{|l|}{ Purpurin } & \pm & + \\
\hline \multicolumn{2}{|l|}{ Kóssa } & + & + \\
\hline \multicolumn{2}{|l|}{ Schmorl } & + & + \\
\hline \multicolumn{2}{|c|}{ Kresylechtviolett } & - & - \\
\hline \multicolumn{2}{|c|}{ Nat. alizarinsulforicum } & + & + \\
\hline \multicolumn{2}{|l|}{ Roehl } & + & - \\
\hline \multicolumn{2}{|c|}{ A. Schultze'sche Reak. } & - & - \\
\hline \multicolumn{2}{|c|}{ Golodetz'sche Reak. } & - & - \\
\hline \multicolumn{2}{|c|}{$\begin{array}{l}\text { Durch Aetheralkohol mit Zusatz von } \\
\text { Salzsäure nach Fischler (Aetheralkohol: } \\
\text { Salzsäure }=100: 1 \text { ) }\end{array}$} & entfärbt & entfärbt \\
\hline \multicolumn{2}{|c|}{$\begin{array}{l}\text { Durch } 1 \% \text { wässerige Salzsäurelösung } \\
\text { nach Fischler }\end{array}$} & entfärbt & entfärbt \\
\hline
\end{tabular}

Fettsäuren. Der Kalksalz in der Rinde besteht aus Phosphatkalk, und solcher im Mark aus fettsaurem Kalk.

5. Haut

In Hämatoxylin-Eosin-Präparaten war die Oberfläche der Epidermis, insbesondere die Hornschicht braungelblich gefärbt und durchsichtig. Die untere Schicht war mit Hämatoxylin homogen und intensiv gefärbt. In der Epidermis fanden sich ofters Vakuolen vor. Im Corium waren reichlich Nadelkristalle. Obwohl das Bindegewebe noch zu erkennen war, waren die Drüsen und Zellen nicht mehr nachweisbar. Durch Nilblausulfat wurde die Hornschicht bräunlich, die Stachelzellenschicht schwach gelblich und das Corium schwach blau gefärbt. 
Durch Sudan III war die Hornschicht gelblichbräunlichrot, die Stachelzellenschicht schwach gelblichrot und das Corium schwach gelblichrot darzustellen. Histochemisch waren geringe Menge Cholesterin-Kephalin und fettsauren Kalks im Corium und Phosphatsalz in den Horn- und Stachelzellenschichten nachzuweisen. Das Resultat ist in Tabelle $\mathrm{V}$ zusammengefasst.

Tabelle V

\begin{tabular}{|c|c|c|c|c|}
\hline \multicolumn{2}{|c|}{ Färbungsmethode } & $\begin{array}{l}\text { Stratum } \\
\text { corneum }\end{array}$ & $\begin{array}{l}\text { Stratum } \\
\text { germinativum }\end{array}$ & $\begin{array}{l}\text { Stratum } \\
\text { corium }\end{array}$ \\
\hline \multicolumn{2}{|l|}{ Nilblausulfat } & gelblichbraun & $\begin{array}{l}\text { schwach } \\
\text { gelblich }\end{array}$ & $\begin{array}{l}\text { schwach } \\
\text { blau }\end{array}$ \\
\hline \multicolumn{2}{|l|}{ Sudan III } & $\begin{array}{l}\text { gelblich- } \\
\text { bräunlichrot }\end{array}$ & $\begin{array}{l}\text { schwach } \\
\text { gelblichrot }\end{array}$ & $\begin{array}{l}\text { schwach } \\
\text { gelblichrot }\end{array}$ \\
\hline \multicolumn{2}{|l|}{ Fischler } & - & - & + \\
\hline \multicolumn{2}{|l|}{ Smith-Dietrich } & - & - & \pm \\
\hline \multicolumn{2}{|l|}{ Ciaccio } & - & - & + \\
\hline \multicolumn{2}{|c|}{ Kresylechtviolett } & - & - & - \\
\hline \multirow{2}{*}{ Osmiumsäure } & I-Reak. & - & - & - \\
\hline & II-Reak. & - & - & - \\
\hline \multicolumn{2}{|l|}{ Purpurin } & + & + & + \\
\hline \multicolumn{2}{|c|}{ Nat. alizarinsulforicum } & + & + & + \\
\hline \multicolumn{2}{|l|}{ Kóssa } & + & + & + \\
\hline \multicolumn{2}{|l|}{ Schmorl } & + & + & + \\
\hline \multicolumn{2}{|c|}{ A. Schultze'sche Reak. } & - & - & - \\
\hline \multicolumn{2}{|c|}{ Golodetz'sche Reak. } & - & - & - \\
\hline \multicolumn{2}{|l|}{ Roehl } & + & + & - \\
\hline \multicolumn{2}{|c|}{$\begin{array}{l}\text { Durch Aetheralkohol mit } \\
\text { Zusatz von Salzsäure nach } \\
\text { Fischler (Aetheralkohol: } \\
\text { Salzsäure=100:1) }\end{array}$} & & & entfärbt \\
\hline \multicolumn{2}{|c|}{$\begin{array}{l}\text { Durch } 1 \% \text { wässerige } \\
\text { Salzsäurelösung nach Fischler }\end{array}$} & & & entfärbt \\
\hline
\end{tabular}


Tabelle VI

\begin{tabular}{|c|c|c|c|c|c|c|}
\hline \multirow{2}{*}{ Material } & \multicolumn{2}{|c|}{$\begin{array}{l}\text { Doppelbrechende } \\
\text { Kreuzfigur }\end{array}$} & \multirow{2}{*}{$\begin{array}{l}\text { Myelin- } \\
\text { bildung }\end{array}$} & \multicolumn{2}{|c|}{ Nadelkristalle } & \multirow{2}{*}{$\begin{array}{l}\text { Die Arten der doppel- } \\
\text { brechenden Substanzen }\end{array}$} \\
\hline & $\begin{array}{l}\text { Bei Er- } \\
\text { hitzung }\end{array}$ & $\begin{array}{l}\text { Bei Ab- } \\
\text { kühlung }\end{array}$ & & $\begin{array}{c}\text { Bei Er- } \\
\text { hitzung }\end{array}$ & $\begin{array}{l}\text { Bei Ab- } \\
\text { kühlung }\end{array}$ & \\
\hline Knochengewebe & + & & - & - & + & $\begin{array}{l}\text { Chloesterin-Palmitinsäure- } \\
\text { ester und Fettsäure- } \\
\text { kristalle }\end{array}$ \\
\hline Hirngewebe & - & + & + & - & + & $\begin{array}{l}\text { Cholesterin, Cholesterin- } \\
\text { Ölsäureester und Fett- } \\
\text { säurekristalle }\end{array}$ \\
\hline Muskel (C) & + & & -- & - & + & $\begin{array}{l}\text { Cholesterin-Palmitinsäure- } \\
\text { ester und Fettsäure- } \\
\text { kristalle }\end{array}$ \\
\hline Muskel (D) & + & & - & - & + & $\begin{array}{l}\text { Cholesterin-Palmitinsäure- } \\
\text { ester und Fettsäure- } \\
\text { kristalle }\end{array}$ \\
\hline Muskel (E) & - & + & - & - & + & $\begin{array}{l}\text { Cholesterin-Ölsäureester } \\
\text { und Fettsäurekristalle }\end{array}$ \\
\hline Muskel (F) & - & + & - & -- & + & $\begin{array}{l}\text { Cholesterin-Ölsäureester } \\
\text { und Fettsäurekristalle }\end{array}$ \\
\hline Muskel (K) & - & + & - & -- & + & $\begin{array}{l}\text { Cholesterin-Ölsäureester } \\
\text { und Fettsäurekristalle }\end{array}$ \\
\hline Muskel (L) & + & & - & - & + & $\begin{array}{l}\text { Cholesterin-Palmitinsäure- } \\
\text { ester und Fettsäure- } \\
\text { kristalle }\end{array}$ \\
\hline Muskel (M) & + & $\begin{array}{l}\mathrm{z} \cdot \mathrm{T} \cdot(-) \\
\mathrm{z} \cdot \mathrm{T} \cdot(+)\end{array}$ & -- & - & + & $\begin{array}{l}\text { Cholesterin-Palmitinsäure- } \\
\text { ester, Cholesterin- } \\
\text { Olsäureester und } \\
\text { Fettsäurekristalle }\end{array}$ \\
\hline Niere & + & & - & - & + & $\begin{array}{l}\text { Cholesterin-Palmitinsäure- } \\
\text { ester und Fettsäure- } \\
\text { kristalle }\end{array}$ \\
\hline Haut & + & & - & - & + & $\begin{array}{l}\text { Cholesterin-Palmitinsäure- } \\
\text { ester und Fettsäure- } \\
\text { kristalle }\end{array}$ \\
\hline
\end{tabular}




\section{UEBER DIE BEZIEHUNGEN ZWISCHEN DER TEMPERATUR UND OPTISCHEM VERHALTEN VON DOPPELBRECHENDEN SUBSTANZEN IN VERSCHIEDENEN GEWEBEN DES LEICHENWACHSES}

Um die Beziehungen zwisshen der Temperatur und optischem Verhalten von doppelbrechenden Lipoiden in fünf Gewebesarten, d.h. in Knochen, Gehirn, Muskel, Niere und Haut zu untersuchen, hat der Verfasser auch Polarisationsmikroskopie ausgeführt und die Lipoidarten nach IDA bestimmt. Er hat darauf hingewiesen, daß Cholesterin-Ölsäureester seinen doppelbrechenden Charakter nur bei niedriger Temperatur beibehält, während Cholesterin und Cholesterinfettsäuregemisch auch bei hoher Temperatur über $100^{\circ} \mathrm{C}$ noch immer doppelbrechend bleiben und Cholesterin-Palmitinsäureester soine Doppelbrechung zwischen $70^{\circ} \mathrm{C}$ und $100^{\circ} \mathrm{C}$ verliert. Nicht nur histologische Färbungen und histochemische Reaktionen, sondern auch Untersuchungen auf Doppelbrechung, insbesondere in Zusammenhang mit der Temperatur, sind in der Bestimmung der Lipoidarten im Gewebe wichtig. Von einer ausführlichen Beschreibung der Resultate wird Abstand genommen, und es sei nur auf die zusammenfassende Darstellung in Tabelle VI hingewiesen.

\section{DIE ENTSTEHUNGSMECHANISMEN DES LEICHENWACHSES}

Da meine Untersuchungen einige Resultate ergeben haben, die zum Verständnis der Leichenwachsentstehung etwas beitragen werden, möchte ich meine Ansicht über die Entstehungsmechanismen des Leichenwachses zusammenfassen, indem ich die Mitteilungen anderer Forscher mit in Erwägung ziehe. Es ist bekannt, daß die Leiche unter gewissen Bedingungen, insbesondere im Wasser, das nur mangelhaft mit der Luft in Berührung kommt, oder in feuchter Erde, nach einer Reihe von chemischen Vorgängen schließlich Leichenwachs entstehen läßt. Die Oberfläche des Leichenwachsos ist makroskopisch grob granuliert und sieht wie rohe Seife aus. Die Meinungen in den Literaturen über die Entstehungsweise des Leichenwachses lassen sich in den folgenden drei Gruppen unterteilen.

1) Fettsäuren und ihre Verbindungen im Leichenwachs entstehen als Dekompositionsprodukte von. Leichenfett.

2) Sie entstehen aus Eiweißkörper der Leiche.

3) Sie können sowohl aus Fett, als auch aus Eiweißkörper der Leiche entstehen.

Unter den Forsshern, die die erste Ansicht vertreten, behauptet THouret, daß keine Fettneubildung im Laufe von Leichenwachsbildung stattfindet. Nach Chevreul, Gunz, Oreilla und Lesieur entsteht das Leichenwachs durch Verbindung von Fettsäuren, die durch Fäulnis von Leichenfett abgegeben 


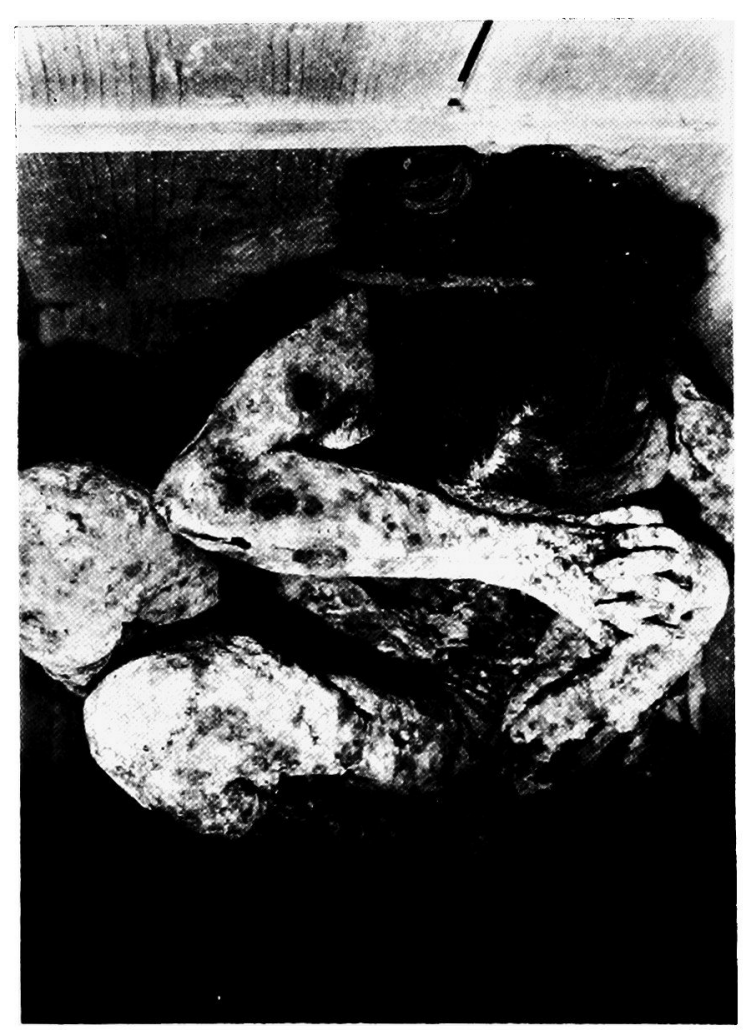

ABBILDUNG 1. Das Gesamtbild der Mumie.

werden, mit Ammoniak, das von Leicheneiweiß herkommt. Wertherill ist der Ansicht, daß sämtliches Neutralfett der Leiche in Glycerin und Fettsäuren abgespalten wird, und dabei entstehende Stearin- und Palmitinsäuren durch Verbindung mit Ammoniak und Kalk Leichenwachs bilden, während ein Teil von Leichenfett mit Eiweißfäulnis vollständig zersotzt wird. Auch vertreten v. Hoffmann, Erman, Reinhardt, Tammassia und Nencki die Ansicht, daß das Leichenwachs nicht aus Bestandteilen von Eiweißkörpern entsteht, sondern aus subkutanem Fettgewebe und aus Fett der sonstigen Gewebe, das trotz fortgeschrittenen Zerfalls anderer Gewebeskomponenten relative gut erhalten bleibt. Der Zerfall von Eiweiß beteiligt sich als solcher nicht an der Bildunng von Leichenwachs. Vielmehr wird der Eiweißkörper völlig durch Fäulnis zersetzt, und dabei wird Glyzerin, das von Fett abgegeben wird, auch mit abgespalten. Die zurückbleibenden Fettsäuren verbinden sich mit Alkali- und Erdalkalimetallen und umwandeln sich schließich ins Leichenwachs (LEBEDEFF). Im Laufe von Fäulnisprozessen kann eine Lageverschiebung von Fett vorkommen, und das Fett tritt auch an der Stelle auf, wo eigentlich kein Fett vorhanden sein 
soll. Solches Fett wird gleichzeitig mit ortsässigem Fett in Glycerin und Fettsäuren abgespalten. Flüssige Ölsäuren und Glycerin werden schon bei mäßig hoher Temperatur zersetzt, und nur die zurückbleibenden höheren Fettsäuren spielen eine wichtige Rolle in Leichenwachsbildung, indem sie durch Verbindung mit Calcium und Magnesium eine Art Seife entstehen lassen (Zillner). Das Leichenwachs entsteht aus vorrätigem Fett des Körpers. Das Muskelgewebe und der Eiweißkörper beteiligen sich nicht an der Bildung des Leichenwachses. Wenn die Leiche in feuchter und von der Luft abgesetzter Erde bleibt, so wird der Eiweißkörper wegen Sauerstoffmangels vollständig autolytisch abgespalten. Dabei entstehendes Ammoniak geht mit gleichzeitig durch Lipase von Fett abgegebene Fettsäuren in Verbindung. Zuerst entstehen dadurch lösliche Seifen, die sich später unter der Wirkung von Calcium und Magnesium in der Erde in unlösliche Formen umwandeln. Da keine Blutzufuhr existiert, kann das Glycerin nicht mehr mit Fettsäuren zu Neutralfett synthetisiert werden, und die Fettsäuren werden kondensiert und scheiden sich schließlich in Kristallen ab (T. Mita).

Die zweite Ansicht wird von folgenden Forschern vertreten. Nach Fourcroy geht das Ammoniak aus dem Stickstoff von Leicheneiweiß mit ebenfalls von Eiweißkörpern abgespaltenen Fettsäuren in Verbindung und läßt Seifen und somit das Leichenwachs entstehen. Die Hauptbestandteile des Leichenwachses bestehen aus Spaltprodukten von Muskeleiweiß (GIBBEs, QUALIN, Virchow). Sie bestehen nicht aus Leichenfettkomponenten, sondern aus von Eiweiß entstandenen höheren Fettarten (VoIT). Nach histologischen Untersuchungen von KratTer, soll das Leichenwachs aus Eiweißkörper im Muskelgewebe gebildet werden. E. SALKowski und H. Salkowski weisen durch experimentelle Studien darauf hin, daß im Laufe von Fäulnis des Muskels und Serumalbumins höhere Fettsäuren entstehen. E. Vort und K. B. Lehmann machen darauf aufmerksam, daß der Fettsäuregehalt in von Fettgewebe befreitem Muskelgewebe zunimmt, wenn es in Wasser bleibt.

Die dritte vermittelnde Meinung ist zum Beispiel von Artilo und ASCARELli vertreten. Nach ihnen wird der Hauptbestandteil des Leichenwachses über unbekannte fermentative Vorgänge aus Leicheneiweiß abgeliefert. Sie nehmen aber auch eine Beteiligung von Leichenfett an Leichenwachsbildung an. Aus den oben zitierten Erörterungen ist ersichtlich, daß nicht nur das Fett, das als solches schon in der Leiche vorhanden ist, sondern auch der Eiweißkörper der Leiche, über verschiedene Stufen der Fäulnisvorgänge, Fettsäuren entstehen läßt. Die Fettsäuren übergehen auf einer Seite weiter zu anderen Derivaten, werden aber auf der anderen Seite durch Alkali- und Erdalkalimetalle verseift und bilden schließlch das Leichenwachs. 


\section{ZUSAMMENFASSUNG}

Im vorangehenden Abschnitt bin ich in eine allgemeine Besprechung über die histologischen, besonders histochemischen Befunde des Leichenwachses eingegangen. Nach bis heute veröffentlichten biochemischen Beobachtungen enthält das Leichenwachs in Muskeln und Weichteilen, mit Ausnahme von Drüsen, kristallene oder amorphe Fettsäuren und Seifen, vor allem Ammonium-, Magnesium- und Calciumsalz von Palmitin- und Stearinsäure (T. ITo). Das Leichenwachs enthält außer sogenannten tierischen Fettsäuren $\alpha$-Monostearinsäure, ein Derivat von Stearinsäure (T. Mita). Littan und Marschal. (1917) weisen darauf hin, daß Hydrooxystearinsäure eine charakteristische Komponente des Leichenwachses ist. Die Tatache, daß Fettsäureseifen, Fettsäurekalksalz, Kalksalze, Cholesterin-Palmitinsäureester und Fettsäurekristalle auch durch meine histochemischen und optischen Untersuchungen nachgewiesen worden sind, bestätigt die Ergebnisse von anderen Forschern. Über das Verhältnis zwischen Fäulnisprozessen und Anfärbbarkeit des Gewebes hat NATORI (1924) folgendes mitgeteilt. Unter den parenchymatösen Organen verliert die Leber in der ersten Linie die Färbbarkeit der Zellkerne, dann folgen die Milz, die Lungen und die Nieren in der Geschwindigkeit der Fäulnisprozesse. Somit ist die Niere am widerstandsfähigsten gegen Fäulnis unter den parenchymatösen Organen. Das Muskelgewebe und andere Organe verlieren die Kernfärbbarkeit ungefähr in gleichem Stadium, obgleich die quergestreiften Muskelfasern verhältnismäßig länger erhalten bleiben. Der Muskel fällt dem Bindegewebe gegenüber viel rascher dem Zerfall anheim. In meinen Präparaten wird gut erhaltenes Bindegewebe dargestellt, obwohl die Zellkerne und Muskelfasern nicht mehr nachweisbar sind. In Bezug auf die Erhaltung des Bindegewebes stimmen diese beiden Resultate (Natori und Mitsuki) bis zu einem gewissen Punkt gut miteinander überein. Das Hirngewebe bleibt verhältnismäBig länger erhalten und stellt auch in meiner eigenen Beobachtung überhaupt das widerstandsfähigste Organ gegen die Fäulnis dar. Daß in einem Muskelstück (E) Kalkreaktion versagt hat, ist wahrscheinlich darauf zurückzuführen, daß Kalksalz in diesem Muskelstück wegen mangelhafter Zufuhr von hartem Wasser nicht gebildet worden war. Cholesterinentstehung in allen Organen ist durch Polarisationsmikroskopie deutlich nachzuweisen. Es muß aber betont werden, daß wir ohne Hilfe von Biochemikern durch Polarisationsmikroskopie und histologische Färbungen allein zur Zeit nicht in der Lage sind, zur genügenden Erkenntnis über die Leichenwachsentstehung zu gelangen. Es wird noch lange dauern, bis hinreichende Färbungen gefunden werden, mit denen wir die Leichenwachsentstehung genauer analysieren und verstehen können. 


\section{SCHLUSSBETRACHTUNG}

1) Im Leichenwachs ist das Knochengewebe am besten erhalten.

2) Folgende Substanzen sind durch meine Untersuchungen in Knochen, Gehirn, Muskel, Haut und Niere des Leichenwachses nachgewiesen :

a) fettsaure Seife, b) fettsaurer Kalk, c) Kalksalz, d) Phosphatkalk, e) Phosphatid, f) Cholesterin +Fettsäure (Ölsäure), g) Cholesterinlecithin und h) Cholesterin + Kephalin. In der Bestimmung einzelner Komponenten wird auf KawamuRA's Gruppenreaktion Bezug genommen.

3) Im Bezug auf die Natur der Lipoide in verschiedenen Geweben des Leichenwachses hat die Polarisationsmikroskopie folgende Substanzen nachgewiesen : a) Cholesterin-Palmitinsäureester, b) Cholesterin, c) CholesterinÖlsäureester und d) Fettsäure und Nadelkristalle.

4) Es wird angenommen, daß Cholesterin im Leichenwachs erst nach dem Tode entsteht.

5) Phosphorsaurer Kalk und andere Kalksalze werden aus ins Leichenwachs imbibierten anorganischen Basen (Calcium und Magnesium) und anorganischer Säure (Phosphorsäure) gebildet.

6) Fettsaurer Kalk und fettsaure Seifen im Gewebe werden aus Komponenten des Leichengewebes gebildet.

7) Unter den Eingeweiden sind das Gehirn und die Niere gegen die Fäulnis am widerstandsfähigsten.

8) Die Bindegewebesfasern bleiben länger als die Muskelfasern erhalten.

9) Quantitative Bestimmung von Cholesterin wurde nach BLooRscher Methode vorgenommen. Der Cholesteringehalt im Muskel beträgt durchschnittlich $2.139 \mathrm{~g}$ pro $100 \mathrm{~g}$ Muskelgewebe.

\section{Literatur}

1) Aschoff, L., Zieglers Beitr., 1910, 47, 1.

2) Ascarelli, Vierteljahrschr. f. gerich. Med., 1906, 32, 219.

3) Chalatow, S.S., Anistrope Verfettung im Lichte d. Pathol. d. Stoffwechsels, I. Aufl., 1922.

4) Erman, Vierteljahrschr. f. gerich. Med., 1882, 37, 51, u. 1884, 40, 29.

5) Hoffmann, V., Lehrbuch der gerich. Med., II. Teil, zehnte Aufl., 1923.

6) Hücke, Arch. f. exp. Patholog. u. Pharmak. 1912, 71, 473.

7) Krause, R., Mikroskopische Technik, III. Aufl., 1926, 2, 1295.

8) Kratter, Lehrbuch der gerich. Med., 1912.

9) Schmidt, W.J., Die Bausteine des Tierkörpers.

10) Wacker, L. u. Hücke, W., Arch. f. exp. Patholog. u. Pharmak., 1913, 74, 443.

11) Zillner, V.J., Schr. f. gerich. Med. N.F., 1881, 35, 193.

12) Asada, Ikaijiho (Jap.), 1926, 1667, 1884.

13) Hanazawa, Mitteilungen aus d. pathol. Instit. d. med. Fakultät, Niigata Universität, 1918, Heft 25.

14) Ida, Hokuetsu Igakukai Zasshi (Jap.), 1931, 7, 344.

15) Ida, Mitteilungen aus d. path. Instit. d. med. Fakultät, Niigata Universität, 1931, 
23.

16) Ito, Kyoto Igaku Zasshi (Jap.), 1928, 25.

17) Koganei, Micromethods for Biochemical Quantitative Analyses, III. Aufl., 1929, 180.

18) Katayama, Kokka Igakukai Zasshi (Jap.), 1910, 273, 1460.

19) Kawamura, R. u. Koyama, M., Cbl. f. allg. Patholog. u. pathol. Anat., 1929, 45, 67.

20) Kawamura, R., Nippon Byorigakkai Zasshi (Jap.), 1928, 231.

21) Kawamura, R., Ikai Jiho (Jap.), 1713, 1124.

22) Kominami, M., Lehrbuch đ. gerich. Med., III. Aufl., 1923.

23) Mita, S., Nisshin Igaku (Jap.), 1925, 6, 309.

24) Mita, S., Tokyo Igakukai Zasshi (Jap.), 1908, 22, 1049.

25) Natori, H., Shakai Igaku Zasshi (Jap.), 1924, 444, 167.

26) Natori, H., Nisshin Igaku (Jap.), 1928, 14, 1997.

27) Miyamori, Aichi Igakukai Zasshi (Jap.), 1933, 7, 1227.

28) Miyamori \& Takagi, Tokyo Iji Shinshi, 1933, 9.

29) Mori, Kyoto Igaku Zasshi (Jap.), 1921, 10, 1460.

30) Okawa, Seikai Zasshi (Jap.), 1924, 43, 44.

31) Sudo, Micromethods for Chemical Quantitative Analyses.

32) Shiroki, Shakai Igaku Zasshi (Jap.), 1926, 475, 421.

33) Shiroki, ebenda, 1926, 477, 55.

34) Takahata, T., Biochemische Analyse, I. Aufl., 1927. 